\title{
El Alma De La Reforma De Córdova De 1918 En La Legislación De La Educación Superior Del Ecuador
}

\section{Marcel Oswaldo Méndez Mantuano}

Magister en Gestión Ambiental, Coordinador de la carrera de Procesamiento de Alimentos del Instituto Tecnológico Superior Juan Bautista Aguirre de la ciudad de Daule, Ecuador

\section{Evelyn Carolina Egüez Caviedes}

Magister en Dirección del Talento Humano, Coordinadora de la carrera de Planificación y Gestión del Transporte Terrestre del Instituto Tecnológico

Superior Juan Bautista Aguirre de la ciudad de Daule, Ecuador

\section{Rosa Lilia Plua Merchán}

Ingeniera Agrónoma, Coordinadora de la carrera de Seguridad y Prevención de Riesgos Laborales del Instituto Tecnológico Superior Juan Bautista

Aguirre de la ciudad de Daule, Ecuador

\section{Jennifer Lisenya Mantuano Flores}

Licenciada en Ciencias de la Educación mención Lengua Inglesa y Lingüística, Docente investigadora de la Unidad Educativa Galo Plaza

Lasso, Ciudad de Daule, Ecuador

URL:http://dx.doi.org/10.19044/esj.2019.v15n4p1

\section{Resumen}

La Reforma Universitaria de Córdoba, básicamente idealizaba un cambio en el concepto de la universidad, donde su génesis fue en una etapa del mundo donde las viejas estructuras caían, diseminándose la aurora progresista, y en cuyo diseño ideológico no cabían las palaciegas estructuras heredadas del colonialismo europeo. La juventud cordobesa no se sentía representada por las autoridades universitarias que pugnaban por mantener los privilegios aristocráticos propios de aquella época, está fue la detonante que dio origen a la revuelta estudiantil de 1918, cuyos enunciados de reclamo se promulgaron como estandarte de la juventud americana que anhelaba romper con la hegemonía de estos exiguos privilegiados, que a su criterio impedían el desarrollo de la ciencia, ergo de la sociedad. Para la presente investigación, se aplicó un enfoque cualitativo, para conocer los postulados de la reforma universitaria de 1918, y así contrastarlos con la articulación legal vigente en el Ecuador. Posee un diseño documental, dado que se recopila información de los hechos a través de varios investigadores, y se detallan las normativas legales que son aplicadas a las instituciones universitarias. Su alcance es 
explicativo, ya que se analizan las aproximaciones entre lo deseado y lo planteado. Se concluye que la autonomía universitaria, el cogobierno, las bases para los concursos de méritos y oposición, la libertad de cátedra, la asistencia libre y la democratización para el ingreso, la gratuidad de la enseñanza, la formación docente constante y el extensionismo investigativo, tuvieron eco en la normativa que gobierna a la educación superior del país.

Palabras Claves: Reforma de córdoba, legislación ecuatoriana, educación superior

\section{The Soul of the Reform of Cordoba in 1981 Based on the Law of Higher Education in Ecuador}

\section{Marcel Oswaldo Méndez Mantuano}

Magister en Gestión Ambiental, Coordinador de la carrera de Procesamiento de Alimentos del Instituto Tecnológico Superior Juan Bautista Aguirre de la ciudad de Daule, Ecuador

\section{Evelyn Carolina Egüez Caviedes}

Magister en Dirección del Talento Humano, Coordinadora de la carrera de Planificación y Gestión del Transporte Terrestre del Instituto Tecnológico

Superior Juan Bautista Aguirre de la ciudad de Daule, Ecuador

\section{Rosa Lilia Plua Merchán}

Ingeniera Agrónoma, Coordinadora de la carrera de Seguridad y Prevención de Riesgos Laborales del Instituto Tecnológico Superior Juan Bautista

Aguirre de la ciudad de Daule, Ecuador

\section{Jennifer Lisenya Mantuano Flores}

Licenciada en Ciencias de la Educación mención Lengua Inglesa y Lingüística, Docente investigadora de la Unidad Educativa Galo Plaza Lasso, Ciudad de Daule, Ecuador

\footnotetext{
Abstract

The University Reformation of Córdoba has basically idealized a change in the concept of university education, where its genesis was at a stage of the world where the old structures fell. The progressive aurora was disseminated. In the ideological design, there were no palatial structures inherited from colonialism European. More so, the youths of Cordoba did not feel represented by the university authorities who struggled to maintain the aristocratic privileges of that time. Hence, it was the trigger that gave rise to
} 
the student revolt of 1918. The claims were promulgated as a banner of the American youth who desired to break the hegemony of these privileged meager, which in his opinion prevented the development of science. For the present investigation, a qualitative approach was applied to know the postulates of the university reform of 1918, and thus contrast them with the current legal articulation in Ecuador. It also has a documentary design, given that the information is collected from the facts through various researchers, and the legal regulations that are applied to university institutions are detailed. Its scope is explanatory since the approximations between the desired and the proposed are analyzed. It is concluded that the university autonomy, the cogovernment, the bases for the competitions of merits and opposition, the freedom of chair, the free attendance and the democratization for the entrance, the gratuitousness of the teaching, the constant educational formation, and the investigative extension were echoed in the regulations that governed higher education in the country.

Keywords: Reform of Cordoba, Ecuadorian legislation, Higher education

\section{INTRODUCCIÓN}

La universidad ecuatoriana tuvo sus orígenes en la conquista europea del continente americano, durante esta etapa se fundaron tres principales centros universitarios, en el año de 1603 se instauró la "Universidad de San Fulgencio", siendo posteriormente reemplazada por "La Real Pontificia Universidad de San Gregorio Magno" en 1622, y en año de 1688 la "Universidad Santo Tomás de Aquino" empieza sus actividades académicas; para el año de 1788 se fusionaron estos dos últimos establecimientos y se crea "La Pública y Real Universidad de Santo Tomás de Aquino", la misma que tenía como misión mejorar y ampliar los estudios existentes. Todas estas instituciones estaban administradas por la curia eclesiástica, quienes mantenían un inflexible control sobre todos los aspectos que circundaban la labor universitaria, aunque de manera nominal estas eran "autónomas", sin embargo respondían al estado regente, el español (Pontificia Universidad Católica del Ecuador, 2011).

En el período republicano se inicia un expansionismo de los centros universitarios, creándose en primeras instancias las universidades para los departamentos de la Gran Colombia en el año 1826, donde el mismo Bolívar redactó las normas de autonomía y de admisión de estudiantes. Posteriormente con la separación del Ecuador, se inicia la época presidencialista, en donde se puede destacar: la expedición del "Decreto Orgánico de Enseñanza Pública" (1836), la "Ley de Estudios" (1853), la creación de las Universidades de Cuenca y Guayaquil (1867), la fundación de la Escuela Politécnica Nacional 
(1869), la eliminación de la autonomía (1880) y la instauración del laicismo (1895) (Pontificia Universidad Católica del Ecuador, 2011; Romero, 2002).

Posteriormente a la revolución liberal de 1895, empiezan a acelerarse las reformas en el ámbito educativo universitario, teniéndose como referencial los manifiestos esgrimidos por los estudiantes de la ciudad de Córdova (Argentina) en el año 1918. No fue trivial el aporte ideológico aportado por las manifestaciones cordobesas, originadas por la reticencia que generaba el sistema educativo de aquella época.

A partir de este hecho histórico, los paradigmas educativos universitarios tomarían un matiz diferente, dado que la chispa revolucionaria de la juventud cordobesa, se esparció sobre el polvorín de las inconformidades que imperaba en las instituciones académicas de toda la América Latina. En este punto álgido convergen dos estructuras antagónicas entre sí, la primera anhelaba mantener el oscurantismo que caracterizó al yugo español sobre sus colonias y donde se percibían las estructuras académicas y de autoridad de las universidades de la era napoleónica (Tünnermann, 2008); mientras que la otra idealizaba una universidad más abierta a los cambios que se erigían como consecuencia natural de la post primera guerra mundial. Estas ideas germinaron en los jóvenes de la Universidad de Córdoba, quienes tuvieron influencias demoliberales y pacifistas de la corriente wilsoniana, con denotada popularidad por los años de 1918 y 1919, sin menoscabar las posturas contra la burguesía y anticlerical (Acevedo, 2011).

A partir de los hechos de la revuelta y posterior reforma universitaria que inició en 1918 en la ciudad de Córdoba, por primera vez se empieza a cuestionar el arcaico modelo universitario ecuatoriano, y dentro del lapso de 1922 a 1925 se alcanzaron importantes victorias de parte de los estudiantes, entre las que se recalcan: la autonomía, el cogobierno, la participación estudiantil en las elecciones de las facultades, la constitución de las federaciones estudiantiles y libertad de cátedra, entre otras (CRESALC, 1986).

En las posteriores décadas se va fortaleciendo la modernización de las instituciones universitarias ecuatorianas, teniéndose como resultado la ampliación de la oferta y demanda para estudios superiores, la proliferación de nuevas universidades, creación de carreras con pertinencia geográfica y la realización de varias reformas a los estatutos universitarios, entre los que destaca el cogobierno, autonomía, libertad de cátedra, financiamiento, entre otros (Ruiz, Torres, \& García, 2017; CRESALC, 1986).

Los postulados que evocaba la Reforma de Córdoba quedaron plasmados en los diferentes estatutos orgánicos que rigieron y rigen en la actualidad a la educación superior del país; ya que los reclamos estudiantiles tuvieron repercusión en la concepción de la humanización y democratización de las universidades, donde se buscaba la congruencia en las nuevas correlaciones económicas, sociales y políticas, dadas las divergencias de las 
nuevas potencias mundiales que polarizaron al mundo en dos ejes (Ruiz, 2002).

Las premisas presentadas en el presente artículo, analiza los postulados ideológicos de los reformistas y los contrasta con las leyes actuales; con el objetivo de esclarecer si los principios anhelados por la juventud de inicios del siglo XX se mantienen vigentes, o si es necesario interiorizar en los preceptos originales y con ello redimir los ideales de aquella juventud que se levantó para acabar con los males de este siglo, que ya no son el rigor de un odio forastero, sino la vergonzosa tiranía del olvido y la indiferencia.

\section{METODOLOGÍA}

El enfoque de la investigación es cualitativa, ya que no se realiza un análisis de datos numéricos o estadísticos, sino que explora desde la subjetividad particular de los autores, aquellos postulados erigidos en la reforma universitaria de Córdoba.

El diseño es de tipo documental, dado que las premisas obtenidas fueron de varios autores que documentaron e interpretaron los reclamos de la juventud cordobesa, además, se analizaron las normativas: Constitución de la República del Ecuador 2008, Ley Orgánica de Educación Superior (LOES), Reglamento de Carrera y Escalafón del Profesor Investigador del Sistema de Educación Superior, y Reglamento de Régimen Académico (RRA); las mismas que tiene jurisdicción vigente en el Ecuador.

El alcance de la investigación es explicativa, donde se muestran las causas que originaron el descontento estudiantil, y como el dogmatismo universitario se mantiene lejos de los reclamos, provocando los enfrentamientos entre dos posturas antagónicas de difícil conciliación. También se especifican los paralelismos ideológicos entre los postulados de los reformistas, y las leyes que rigen a la educación superior en el Ecuador.

\section{DESARROLLO}

\section{Origen del descontento cordobés}

Desde el año de 1917 los estudiantes vinculados a la asociación de "Córdoba Libre", mantenían discrepancias con las autoridades de la Universidad Nacional de Córdoba, sus reclamos estaban orientados a renovar la planta docente que era vitalicia y muy arraigada a las costumbres católicas, que en su visión (de los estudiantes), el férreo dogmatismo religioso impedía la instauración de nuevas ideas que mimeticen con el pragmatismo industrial que se encontraba en desarrollo, también esbozaban el rediseño en la estructura administrativa universitaria, la misma que se sometía a los poderes eclesiásticos o políticos de turno. El crecimiento de las inconformidades de parte del estudiantado generó en la creación del comité Pro Reforma Universitaria, quienes representarían a toda una sociedad emergente que se 
identificaría con los fundamentos renacentistas del nuevo siglo. Entre las primeras medidas tomadas por el comité, fue convocar a una huelga estudiantil. Los dirigentes buscaron alianzas con los movimientos de otras zonas de la República Argentina, con el objetivo de enlazar una red de apoyo para oponerse al dominio educativo católico y en pro de la defensa de una cultura liberal (Bustelo, 2014).

Los reformistas mantienen la unidad, mientras van sumando el apoyo de diferentes sectores de la sociedad, en abril de 1918 se crea la Federación Universitaria de Argentina, siendo conformada por estudiantes de Tucumán, Santa Fé, Córdoba, La Plata y Buenos Aires. La respuesta al levantamiento fue la esperada, las autoridades reprimen y menoscaban a todo aquel que intente levantarse contra el "orden instituido", quienes en un intento de calmar la ebullición ascendente de los ánimos, convocan a elecciones de autoridades para el 15 de junio; las mismas fueron minimizadas por los estudiantes, quienes expresaron que fueron fraudulentas y en beneficio de los jesuitas (ejercían el poder de facto en la universidad) y no las reconocieron como legitimas (Acevedo, 2011).

El 21 de junio se proclama el Manifiesto Liminar (previa declaratoria de huelga indefinida), que apaleaba al idealismo revolucionario con connotaciones utópicas de romanticismo ideológico (Samacá \& Acevedo, 2011).

Las manifestaciones de fuerza aumentaban con el trascurrir de los días, hasta llegar a varias intervenciones de la Universidad; además de pedir que la fuerza pública impidiera la entrada a los estudiantes (de parte de los tradicionalistas), dadas las violentas irrupciones de estos para impedir que alguien con una postura divergente (a la de ellos) ejerciera la rectoría. La disidencia estudiantil provocó que se llevara a cabo en Córdoba el Primer Congreso Nacional de Estudiantes entre el 20 y el 31 de julio de 1918, convocada por las principales federaciones estudiantiles de Argentina (Buenos Aires, Córdoba, La Plata, Tucumán y Litoral). En el congreso se analizaron nuevas leyes y estatutos sobre autonomía universitaria, gobierno tripartito paritario, asistencia libre, docencia libre, bienestar estudiantil, libertad de juramento y nacionalización de las universidades provinciales del Litoral y Tucumán. En este mismo espacio se declaró al 15 de junio como el día de "La Reforma" (Caldelari, Tcach, \& Brav, 2008).

\section{Postulados de la Reforma}

Varios autores han estudiado los postulados de los reformitas cordobeses, los mismos que fueron compilados de los sucesivos manifiestos (principalmente el Manifiesto Liminar) y de los discursos durante las revueltas estudiantiles. La siguiente lista se basa en la realizada por Tünnermann (2008), 
quien resume los reclamos de los estudiantes cordobeses de la siguiente manera:

1. Autonomía universitaria, que comprendía lo político, la docencia, lo administrativo y lo económico. Con ello se deseaba evitar la injerencia del estado para subordinar al estudiantado; sin embargo, se reclamaba del mismo, los recursos económicos necesarios para la subsistencia de la universidad.

2. Cogobierno, o elección de los directivos y de las autoridades de la universidad por la propia comunidad universitaria, con la participación de docentes, estudiantes y graduados.

3. Concursos de oposición para la selección del profesorado, y que estos tengan un período de tiempo en la impartición de las cátedras.

4. Docencia libre o libertad de cátedra, para que exista la posibilidad de que toda persona con capacidad y vocación docente pueda incorporarse a la universidad, sin tener la obligación de seguir un orden dogmático preestablecido, a fin de asegurar la pluralidad ideológica.

5. Asistencia libre y democratización del ingreso, para que exista una flexibilización en las jornadas académicas, de tal manera, que se permita a la clase obrera desempeñarse como estudiantes y trabajadores de manera paralela; además para que nadie tenga privilegios en los ingresos, y de esta manera sean excluidas personas talentosas.

6. Gratuidad de la enseñanza, para aproximar los conocimientos a los diferentes estratos sociales, y que no sea de exclusividad para algunos privilegiados.

7. Reorganización académica, creación de nuevas escuelas y modernización de los métodos de enseñanza; es decir una docencia activa, que consienta el continuo mejoramiento de la formación de los profesionales que se integran en las aulas universitarias.

8. Vinculación con el sistema educativo nacional, que exista una simbiosis efectiva con los otros niveles de enseñanza previos.

9. Extensión universitaria, fortalecimiento de la función social de la universidad, para lograr que la universidad se involucre activamente en la investigación, para la posterior solución de problemas de la sociedad y así contribuir al bienestar de la nación.

10. Unidad latinoamericana, para buscar la integración con otras universidades y con ello luchar contra todas las formas de autoritarismos.

\section{Los postulados reformistas en los articulados legales de Ecuador}

En la actualidad existen cuatro cuerpos legales que norman las principales acciones de la educación universitaria en el Ecuador, los cuales son: Constitución de la República del Ecuador 2008, Ley Orgánica de Educación Superior (LOES), Reglamento de Carrera y Escalafón del Profesor 
Investigador del Sistema de Educación Superior y Reglamento de Régimen Académico (RRA).

La autonomía universitaria está plasmada en la Constitución del Ecuador en sus artículos 3, 351 y 355, en los cuales se establece que:

"Art. 355.- El Estado reconocerá a las universidades y escuelas politécnicas autonomía académica, administrativa, financiera $y$ orgánica, acorde con los objetivos del régimen de desarrollo y los principios establecidos en la Constitución”.

Esta autonomía debe ser sostenida de una manera solidaria y responsable, con el objetivo de buscar la verdad sin ningún tipo de prohibiciones, donde deben coexistir los principios de alternancia y transparencia. También se instituye la inviolabilidad de los recintos universitarios, donde el orden interno será de única responsabilidad de las autoridades universitarias, y únicamente bajo autorización expresa se facultará el ingreso de la fuerza pública. La autonomía universitaria también conlleva una fiscalización de parte los entes pertinentes, es decir, no se exime de responsabilidad por la mala administración de los recursos públicos. Los fondos presupuestarios para las actividades académicas y administrativas están garantizados de parte del Estado ecuatoriano.

En la Ley Orgánica de Educación Superior (LOES), en sus artículos $12,13,17$ y 18 se subraya este principio, dando detalles específicos de lo que comprende esta autonomía responsable (art. 18), teniendo entre los aspectos más relevantes: la independencia de los docentes para la libertad de cátedra; libertad para que la universidad puedan expedir estatutos, planes y programas de estudios; libertad para nombrar a las autoridades, docentes, investigadores, servidores y trabajadores; libertad para gestionar procesos internos; libertad para la realización de los presupuestos; libertad de la administración del patrimonio y de los recursos de la universidad (en concordancia con la Ley); libertad para determinar sus formas y órganos de gobierno (en consonancia con la Ley). En el artículo 24, se establece que los recursos económicos para cada una de las universidades estará en función del número de estudiantes, de la experiencia de los docentes, tipología de la institución, así como de sus carreras y programas de estudios, vinculación con la sociedad, eficiencia en docencia e investigación, eficiencia terminal y administrativa; conjuntamente se instaura que bajo ningún concepto se despojará a las instituciones de este derecho.

A la par, la Constitución de la República en su artículo 351, nombra al cogobierno como uno de los principios en los cuales se sustenta el sistema de educación superior, de igual forma en la LOES en el Título III (capítulos 1 y 2 ), se establecen los principios y estatutos para la conformación de los cogobiernos en las universidades y escuelas politécnicas públicas o privadas. Dentro de lo que se destaca lo siguiente: el cogobierno es la parte consustancial 
de la autonomía universitaria; el cogobierno es la dirección y administración compartida de la universidad y escuelas politécnicas, de parte de los diferentes sectores que la integran (profesores, estudiantes, empleados y trabajadores); deberán existir un órgano colegiado de carácter académico y administrativo, cuya función específica estará demarcada en los estatutos y reglamentos de cada institución; el órgano colegiado será la máxima autoridad dentro de los establecimientos educativos y estará conformado por las autoridades y representantes de los profesores, estudiantes y graduados; se establecen requisitos para las funciones de rector y vicerrector; se delimitan el tiempo para el cargo de rector y vicerrector por un lapso de 5 años; se instaura la paridad de género, igualdad de oportunidades y equidad; la votación de los estudiantes para la elección de rector o vicerrector equivaldrá entre un $10 \%$ al $25 \%$; la votación de los servidores y trabajadores para la elección de rector o vicerrector equivaldrá entre un $1 \%$ al $5 \%$; estos porcentajes también se aplican para la elección del órgano colegiado y adicionalmente se agrega la votación de los graduados para la elección, la cual equivaldrá entre un $1 \%$ al $5 \%$.

La LOES en los artículos 13 h, 91, 150 y 152, contemplan el concurso de méritos y oposición para aquellos docentes que deseen ingresar a las instituciones de educación superior. En el Reglamento de Carrera y Escalafón del Profesor Investigador del Sistema de Educación Superior, se dan las especificaciones necesarias para el ingreso de docentes por medio de los concursos públicos de méritos y oposición, entre lo que se destaca: el personal de las instituciones de educación superior se clasifican como titulares y no titulares, los titulares son aquellos que ganan el respectivo concurso (art. 5); las instituciones de educación superior podrán llamar a concursos para las vacantes de técnicos docentes, técnicos de investigación, técnicos de laboratorio, ayudantes de docencia y de investigación, y técnicos en el campo de las artes o artistas docentes (art. 19, 20, 21, 22, 23); asimismo las universidades, escuelas politécnicas y los institutos superiores técnicos, tecnológicos, pedagógicos, de artes y conservatorios superiores, realizarán los concursos para el ingreso de personal académico titular auxiliar, personal académico titular agregado, personal académico titular principal y personal académico titular principal investigador (art. 30, 31, 32, 33, 38, 39, 40).

En el artículo 44 del reglamento anteriormente citado, se establecen las etapas para la realización del concurso de méritos y oposición, entre las que se encuentran: la fase de méritos, la cual consiste en el análisis, verificación y calificación de los documentos que presentan aquellos docentes postulantes; la fase de oposición, que consta de pruebas teóricas y prácticas tanto orales como escritas, así como la exposición de un proyecto de investigación, creación o innovación (no es obligatorio para los postulantes de personal académico titular auxiliar 1 o titular agregado 1). Este mismo artículo establece que la fase de oposición, deberá tener un peso de entre $50 \%$ a $75 \%$ 
del total de la calificación del concurso para profesores e investigadores auxiliares y agregados; y entre $30 \%$ y $70 \%$ para profesores e investigadores principales. Las instituciones que realizarán los concursos difundirán las convocatorias en por lo menos dos medios de comunicación masivos, así como en su web institucional y las redes electrónicas establecidas por el organismo del ramo pertinente.

Coexiste la garantía para que los docentes puedan ejercer la libertad de cátedra, la que comprende de parte de las instituciones y docentes, en la exposición más adecuada (de acuerdo a su criterio) de los contenidos de los programas de estudios, de igual manera, la libertad investigativa asiente en buscar la verdad en los distintos ámbitos científicos, sin ningún tipo de obstáculos, exceptos los previstos en las leyes (art. 146 del LOES).

En el capítulo VI del RRA, se establecen diferentes modalidades de estudio, las cuales son: presencial, semipresencial, dual, en línea y a distancia; las que permiten a los estudiantes establecer con anterioridad las singularidades que significa estudiar una carrera profesional, considerando los horarios adecuados de acuerdo a sus demás actividades, sin que exista algún tipo de imposición para continuar con una determinada jornada de estudios.

En el artículo 356 de la Constitución, se menciona que la educación superior será gratuita hasta el tercer nivel, adicionalmente se avala la igualdad de oportunidades en el acceso, pertinencia, movilidad y egreso de los estudiantes. El examen nacional "Ser Bachiller" para el ingreso a las instituciones superiores públicas del país, garantiza esta igualdad de oportunidades, ya que se evalúa de manera indistinta, el desarrollo de las aptitudes y destrezas que los estudiantes deben alcanzar al culminar el bachillerato y que son indispensables para el desenvolvimiento exitoso como ciudadanos y para poder acceder a estudios superiores (art. 81 LOES). En el artículo 80 de la LOES, también indica que para mantener la gratuidad, dependerá de algunos criterios, entre los cuales están: matricularse en al menos el $60 \%$ de las asignaturas de un determinado nivel académico; inscribirse en el nivel preuniversitario, prepolitécnico o su equivalente; no se cubrirán las segundas ni terceras matrículas, tampoco las consideradas especiales o extraordinarias; el estado financiará una sola carrera o programa del tercer nivel; se pierde de manera definitiva la gratuidad, si un estudiante regular reprueba el $30 \%$ de las materias o créditos de la malla curricular de la carrera cursada.

En la actualidad, las instituciones educativas no solo deben ocuparse de promover la formación y mejorar el desempeño de sus alumnos, sino también de su equipo docente. Considerando la complejidad de la tarea que deben afrontar, el aprendizaje continuo resulta indispensable para que los maestros posean los conocimientos y dispongan de las herramientas necesarias para lograr tal propósito de forma satisfactoria, es por ello, que en el articulado 
legal de educación superior, permite a los docentes actualizar y profundizar en sus conocimientos, habilidades y destrezas para desarrollar la docencia y la investigación con calidad, innovación y alto sentido de compromiso, dando de parte de las instituciones, la flexibilidad e incentivos para ello. La LOES en el artículo 13 y 156, así como en los artículos 90, 91 y 92 del Reglamento de Carrera y Escalafón del Profesor Investigador del Sistema de Educación Superior, reconocen al perfeccionamiento docente como indispensable para los actores del sistema de educación superior, existiendo partidas especiales que son destinadas para financiar planes de becas o ayudas económicas para especialización o capacitación y año sabático.

Actualmente no hay una efectiva coyuntura entre la educación de bachillerato y la superior o universitaria, ya que existen dos órganos rectores que trabajan de maneras aisladas (Ministerio de Educación y la Secretaría de Educación Superior, Ciencia, Tecnología e Innovación), donde convergen leyes específicas para cada uno de ellos, y de esta manera se contribuye a la separación de estas dos entidades. Esta visión se ve reforzada, dado que exclusivamente en el artículo 81 de la LOES, menciona una articulación entre el nivel bachiller o su equivalente y la educación superior pública; sin embargo, en la práctica pocos son los esfuerzos para realizarlo de manera paulatina y efectiva.

La investigación con relación a los problemas de índole local, están abordados en el RRA en sus artículos 74, 75 y 76, los cuales señalan que: en todos los niveles formativos, la investigación deberá ser diseñada en función del contexto social y cultural en la cual se desenvuelve la institución; las investigaciones en redes (nacionales o internacionales) deben guardar correspondencia con los requerimientos, prioridades y propósitos del Plan Nacional de Desarrollo, planes locales, planes regionales y programas internacionales de investigación en los campos de la educación superior, la ciencia, la cultura, las artes y la tecnología; las instituciones propondrán la articulación de estos proyectos de investigación en función a las necesidades de cada territorio, país o región.

La unidad latinoamericana como la aspiraban los reformistas cordobeses, no está plasmada en los lineamientos legales ecuatorianos, ya que esta integración buscaba ser una plataforma de activistas políticos (o hasta injerencista de asuntos nacionales) y no académicas. Sin embargo, en la LOES (art. 138) y el RRA (art. 75, 103) permiten la relación y la creación de redes con instituciones de educación superior internacionales, para permitir la investigación conjunta, la movilidad de docentes, estudiantes e investigadores, vinculación con la comunidad, educación continua, la innovación tecnológica y el diseño e implementación de programas de desarrollo.

El análisis anterior, permite esclarecer los puntos en los cuales convergen los postulados reformistas de 1918 con las leyes ecuatorianas (y en 
cuales no), que a pesar de la constante evolución legal, existen premisas que se han mantenido estables durante diferentes etapas del derecho en el Ecuador; destacándose el espíritu del reclamo de la juventud cordobesa de 1918. Los cuales quedaron plasmados (en su mayoría), en las normativas legales de antaño y que se han reformulado en la actualidad, pero manteniendo la esencia idealista, característica de la juventud inconforme, que anhela erradicar las formas de elitismo, para mantener una universidad que responda a la sociedad y no a los intereses egoístas de los grupos de poder.

Los postulados de 1918, que están normalizados en las leyes y reglamentos del Ecuador, son los siguientes:

- Autonomía universitaria,

- Cogobierno,

- Concursos de méritos y oposición,

- Libertad de cátedra,

- Asistencia libre y la democratización para el ingreso,

- Gratuidad de la enseñanza,

- Formación docente, y

- Extensionismo investigativo.

Sin embargo, también existen otros postulados que no se instaurado o son vagamente mencionados en los cuerpos legales, entre los que destacan:

- Articulación con todos los niveles de enseñanza y

- Unidad latinoamericana.

\section{CONCLUSION}

La Reforma Universitaria de Córdoba inició como un movimiento liberal con tintes ambivalentes, ya que conciliaban lo político como lo académico, y de vocación latinoamericana y antiimperialista.

La mayoría de los postulados propuestos, tardaron décadas en ser implementados en el Ecuador, incluso muchos de ellos son netamente teóricos, ya que en la práctica no son aplicables dado su utopismo.

De manera general: la autonomía universitaria, el cogobierno, los concursos de méritos y oposición, la libertad de cátedra, la asistencia libre y la democratización para el ingreso, la gratuidad de la enseñanza, la formación docente y el extensionismo investigativo; estaban entre los objetivos anhelados por los reformistas cordobeses y están contemplados en los articulados legales del Ecuador.

Sin embargo: la articulación con todos los niveles de enseñanza y la unidad latinoamericana son tareas pendientes por las instituciones de educación superior del país.

Las instituciones de educación superior ecuatorianas, por medio de la ley, tratan de emulan y alcanzar los principios reformistas de 1918, los mismos 
que anhelaban la formación de profesionales con la capacidad de cambiar su entorno y disminuir la desigualdad social, eliminando las formas de elitismo.

La sociedad no debe consentir la enajenación de los laureles del pasado, sino ser observadores de estos derechos, que fueron ganados bajo el filo del acero.

\section{References:}

1. Acevedo, A. (2011). A cien años de la reforma de Córdoba, 19182018. La época, los acontecimientos, el legado. Dialnet. No. 36. Recuperado https://dialnet.unirioja.es/servlet/articulo?codigo=3797042.

2. Bustelo, N. (2014). La Reforma Universitaria desde sus Grupos y Revistas una Reconstrucción de los Proyectos y las Disputas del Movimiento Estudiantil Porteño de las Primeras Décadas del Siglo XX (1914-1928) (Tesis doctoral). Universidad Nacional de la Plata, Argentina.

3. Caldelari, M., Tcach, C., \& Bravo, M. (2008). La Gaceta Universitaria 1918-1919: una mirada sobre el movimiento reformista en las universidades nacionales. Buenos Aires, Argentina: Eudeba.

4. Constitución de la República del Ecuador (2008). Registro Oficial 449, Quito, Ecuador, 20 de octubre del 2008.

5. CRESALC - Centro Regional para la Educación Superior en América Latina y el Caribe (1986). La educación Superior en el Ecuador. Caracas, Venezuela: CRESALC - UNESCO.

6. Ley Orgánica de Educación Superior, LOES. Registro Oficial Suplemento 298, Quito, Ecuador, 12 de octubre del 2010.

7. Pontificia Universidad Católica del Ecuador (2011). Simposio Permanente sobre la Universidad No. 3. Historia de la Universidad en el Ecuador, sus principales hitos. Quito, Ecuador: PPL Impresores.

8. Reglamento de Carrera y Escalafón del Profesor e Investigador del Sistema de Educación Superior (Codificación). RPC-SO-037-No.Z652012, Quito, Ecuador, 13 de julio del 2017.

9. Reglamento de Régimen Académico, RRA. RPC-SO-17-No.2692016, Quito, Ecuador, 04 de mayo del 2016.

10. Romero, N. (2002). Evolución de la Legislación en materia de Educación Superior en Ecuador. Quito, Ecuador: UNESCO.

11. Ruiz, L., Torres, G., \& García, D. (2017). Desafíos de la Educación Superior. Consideraciones sobre el Ecuador. INNOVA Research Journal, 3 (2), 8 - 16 pp.

12. Ruiz, G. (2002). La sociedad del conocimiento y la educación superior universitaria. Revista Mexicana de Ciencias Politicas y Sociales, 45 (185), 109 - 124 pp. 
13. Samacá, G. \& Acevedo, A. (2011). De la reforma de Córdoba al Cordobazo: La universidad como escenario de las luchas por la democracia en Argentina, 1918 - 1969 y su impacto en Colombia. Memorias. Vol. 8 (15), 170 - 195 pp.

14. Tünnermann, C. (2008). 90 años de Reforma Universitaria de Córdoba (1918-2008). Nicaragua: Hispamer. 